ANNALES

POLONICI MATHEMATICI

$95.1(2009)$

\title{
Splitting vector bundles by blowups
}

\author{
by Wojciech Kucharz (Albuquerque, NM)
}

\begin{abstract}
We show some advantages of splitting vector bundles by blowups.
0. Introduction. In topology and algebraic geometry the splitting of vector bundles plays an important role (cf. [5, 8]). Usually, it is based on the following construction. For any vector bundle $E$ on $X$, we have the associated projective bundle $q: \mathbb{P}(E) \rightarrow X$. The pullback vector bundle $q^{*} E$ contains the tautological line bundle $L_{E}$ on $\mathbb{P}(E)$. In algebraic geometry it is sometimes convenient to use instead the pullback by the projection of an appropriate blowup (cf. [9]). We demonstrate below that blowups work very well for smooth vector bundles on smooth manifolds and for algebraic vector bundles on nonsingular real algebraic sets. As an application, we obtain a nice description of the Stiefel-Whitney classes. We consider only real vector bundles.
\end{abstract}

1. Vector bundles on smooth manifolds. All smooth (of class $\mathcal{C}^{\infty}$ ) manifolds are assumed to be paracompact and without boundary. Submanifolds are supposed to be closed subsets of the ambient manifold. The tangent bundle to a smooth manifold $X$ will be denoted by $T_{X}$. If $Z$ is a smooth submanifold of $X, \operatorname{dim} Z<\operatorname{dim} X$, we denote by

$$
\pi(X, Z): B(X, Z) \rightarrow X
$$

the blowup of $X$ at $Z$. Recall that as a point set $B(X, Z)$ is the union of $X \backslash Z$ and the projective bundle $\mathbb{P}\left(N_{Z} X\right)$ on $Z$ associated with the normal bundle

$$
N_{Z} X:=\left(T_{X} \mid Z\right) / T_{Z}
$$

to $Z$ in $X$. The map $\pi(X, Z)$ is the identity on $X \backslash Z$ and the bundle projection $\mathbb{P}\left(N_{Z} X\right) \rightarrow Z$ on $\mathbb{P}\left(N_{Z} X\right)$. On $B(X, Z)$ there is a natural smooth manifold structure, and $\pi(X, Z)$ is a smooth map.

2000 Mathematics Subject Classification: 57R22, 57R20, 14P25.

Key words and phrases: blowups, splitting vector bundles, Stiefel-Whitney classes. 
If $E$ is a vector bundle on $X$ and $s: X \rightarrow E$ is a section, we denote by $Z(s)$ the zero locus of $s$,

$$
Z(s)=\{x \in X \mid s(x)=0\} .
$$

TheOREM 1.1. Let $X$ be a smooth manifold. Let $E$ be a smooth vector bundle on $X$ and let $s: X \rightarrow E$ be a smooth section transverse to the zero section. If $\pi(X, Z): B(X, Z) \rightarrow X$ is the blowup of $X$ at $Z=Z(s)$, then the pullback vector bundle $\pi(X, Z)^{*} E$ on $B(X, Z)$ contains a smooth line subbundle.

Proof. We regard $X$ as a submanifold of $E$, identifying it with its image by the zero section. Moreover, we identify the normal bundle to $X$ in $E$ with $E$. Thus as a point set $B(E, X)$ is the union of $E \backslash X$ and $\mathbb{P}(E)$, while $\pi(E, X): B(E, X) \rightarrow E$ is the identity on $E \backslash X$ and the bundle projection $\mathbb{P}(E) \rightarrow X$ on $\mathbb{P}(E)$. If $p: E \rightarrow X$ is the bundle projection, then the pullback vector bundle $(p \circ \pi(E, X))^{*} E$ on $B(E, X)$ contains a smooth line subbundle $L$ defined as follows. The fiber of $L$ over a point $e$ in $E \backslash X$ is the line $\{e\} \times(\mathbb{R} e)$, and the restriction $L \mid \mathbb{P}(E)$ is the tautological line bundle on $\mathbb{P}(E)$.

Since $s$ is transverse to $X$ in $E$, for every point $z$ in $Z$, the differential $d s_{z}: T_{X, z} \rightarrow T_{E, z}$ induces a linear isomorphism

$$
\bar{d} s_{z}:\left(N_{Z} X\right)_{z} \rightarrow\left(N_{X} E\right)_{z}=E_{z}
$$

between the fibers over $z$ of the normal bundle to $Z$ in $X$ and the normal bundle to $X$ in $E$.

Define $\bar{s}: B(X, Z) \rightarrow B(E, X)$ by $\bar{s}(x)=x$ for all $x$ in $X \backslash Z$ and $\bar{s}(l)=\bar{d} s_{z}(l)$ for all $l$ in $\mathbb{P}\left(\left(N_{Z} X\right)_{z}\right)$ (thus $\bar{s}(l)$ is in $\left.\mathbb{P}\left(E_{z}\right)\right)$. By construction, $\bar{s}$ is a smooth map satisfying

$$
p \circ \pi(E, X) \circ \bar{s}=\pi(X, Z) .
$$

Hence $\bar{s}^{*} L$ is a smooth line subbundle of

$$
\bar{s}^{*}\left((p \circ \pi(E, X))^{*} E\right)=(p \circ \pi(E, X) \circ \bar{s})^{*} E=\pi(X, Z)^{*} E .
$$

The proof is complete.

Corollary 1.2. Let $X$ be a smooth manifold and let $E$ be a smooth vector bundle on $X$ of rank $r+1, r \geq 1$. Then there exists a sequence of smooth maps

$$
\widetilde{X}=X_{r} \stackrel{\pi_{r}}{\longrightarrow} X_{r-1} \stackrel{\pi_{r-1}}{\longrightarrow} \cdots \stackrel{\pi_{2}}{\longrightarrow} X_{1} \stackrel{\pi_{1}}{\longrightarrow} X_{0}=X
$$

such that each $\pi_{i}$ is the blowup of $X_{i-1}$ at a smooth submanifold which is either empty or of codimension $r+2-i, 1 \leq i \leq r$, and the pullback vector bundle $\pi^{*} E$ by the composite map $\pi=\pi_{1} \circ \cdots \circ \pi_{r}$ is a direct sum of smooth line bundles on $\widetilde{X}$. 
Proof. This follows by induction from Theorem 1.1 since every smooth vector bundle has a smooth section transverse to the zero section, and every smooth subbundle is a direct summand.

Corollary 1.2 can be useful in the computation of the $k$ th Stiefel-Whitney class $w_{k}(E)$ of $E, k \geq 0$. Indeed, it should be easy in principle to compute $w_{k}\left(\pi^{*} E\right)$ since $\pi^{*} E$ is a direct sum of line bundles. Moreover, basic topological properties of the map $\pi: \widetilde{X} \rightarrow X$ are well understood: see Corollary 1.3 below. For simplicity we will assume that $X$ is compact.

For any compact smooth manifold $M$ of dimension $m$, we have the Poincaé duality isomorphism

$$
D_{M}: H^{k}(M ; \mathbb{Z} / 2) \rightarrow H_{m-k}(M ; \mathbb{Z} / 2), \quad D_{M}(v)=v \cap[M],
$$

where $[M]$ is the fundamental class of $M$ in $H_{m}(M ; \mathbb{Z} / 2)$.

Corollary 1.3. With notation as in Corollary 1.2 , $\operatorname{dim} \tilde{X}=\operatorname{dim} X$ and $\pi: \widetilde{X} \rightarrow X$ is a proper map. If $X$ is compact, then the induced homomorphism

$$
\pi^{*}: H^{*}(X ; \mathbb{Z} / 2) \rightarrow H^{*}(\widetilde{X} ; \mathbb{Z} / 2)
$$

is injective and

$$
D_{X}\left(w_{k}(E)\right)=\pi_{*}\left(D_{\widetilde{X}}\left(w_{k}\left(\pi^{*} E\right)\right)\right) \quad \text { for all } k \geq 0 .
$$

Proof. It follows directly from the construction that $\operatorname{dim} \widetilde{X}=\operatorname{dim} X$ and $\pi: \widetilde{X} \rightarrow X$ is a proper map. If $X$ is compact, then $\pi_{*}([\widetilde{X}])=[X](\bmod 2$ topological degree of $\pi$ is equal to 1 ) and for every cohomology class $v$ in $H^{k}(X ; \mathbb{Z} / 2)$, we have

$$
D_{X}(v)=v \cap[X]=v \cap \pi_{*}([\widetilde{X}])=\pi_{*}\left(\pi^{*}(v) \cap[\widetilde{X}]\right)=\pi_{*}\left(D_{\widetilde{X}}\left(\pi^{*}(v)\right)\right) .
$$

In particular, $\pi^{*}(v)=0$ implies $v=0$, and hence $\pi^{*}$ is injective.

Taking $v=w_{k}(E)$, we get

$$
D_{X}\left(w_{k}(E)\right)=\pi_{*}\left(D_{\widetilde{X}}\left(\pi^{*}\left(w_{k}(E)\right)\right)\right)=\pi_{*}\left(D_{\widetilde{X}}\left(w_{k}\left(\pi^{*} E\right)\right)\right) .
$$

The proof is complete.

It is not hard to prove that $\pi^{*}$ is injective even if $X$ is not necessarily compact. The equality in Corollary 1.3 is also true in the noncompact case, provided that one uses the Borel-Moore homology instead of the singular homology. A very readable introduction to the Borel-Moore homology is in [4, Appendix B].

2. Vector bundles on nonsingular real algebraic varieties. In this section by a real algebraic variety we mean a locally ringed space isomorphic to a real algebraic set (in $\mathbb{R}^{n}$ for some $n$ ) endowed with the Zariski topology and the sheaf of regular functions. In [2] such objects are called affine 
real algebraic varieties. Subvarieties will be assumed to be Zariski closed in the ambient variety. Morphisms between real algebraic varieties will be called regular maps. Every real algebraic variety also carries the Euclidean topology, that is, the topology induced by the usual metric on $\mathbb{R}$. Unless explicitly stated otherwise, all topological notions related to real algebraic varieties will refer to the Euclidean topology.

Every nonsingular real algebraic variety is in a natural way a smooth manifold. Recall that every Zariski locally closed subset of real projective space $\mathbb{P}^{n}(\mathbb{R})$, with the sheaf of regular functions on it, is a real algebraic variety (cf. [2, Proposition 3.2.10, Theorem 3.4.4]). In particular, if $X$ is a nonsingular real algebraic variety and $Z$ is a nonsingular subvariety of $X$ with $\operatorname{dim} Z<\operatorname{dim} X$, then the blowup $B(X, Z)$ of $X$ at $Z$ is a nonsingular real algebraic variety, and the blowup projection $\pi(X, Z): B(X, Z) \rightarrow X$ is a regular map (cf. [2, Proposition 3.5.11]). For basic properties of algebraic vector bundles on real algebraic varieties the reader may refer to [2]. Let us mention that algebraic vector bundles considered here and in [2] are called strongly algebraic vector bundles in [1].

TheOREM 2.1. Let $X$ be a nonsingular real algebraic variety. Let $E$ be an algebraic vector bundle on $X$ and let $s: X \rightarrow E$ be an algebraic section transverse to the zero section. If $\pi(X, Z): B(X, Z) \rightarrow X$ is the blowup of $X$ at $Z=Z(s)$, then the pullback vector bundle $\pi(X, Z)^{*} E$ on $B(X, Z)$ contains an algebraic line subbundle.

Proof. The same as the proof of Theorem 1.1. It is sufficient to observe that the map $\bar{s}: B(X, Z) \rightarrow B(E, X)$ constructed in the proof of Theorem 1.1 is regular in the case under consideration here.

Every algebraic vector bundle $E$ on a nonsingular real algebraic variety $X$ has an algebraic section transverse to the zero section. In fact, a slightly stronger statement, needed in the proof of Corollary 2.3 below, is true. Consider $X$ as a subvariety of $E$, identifying it with its image by the zero section. If $\mathcal{A}$ is a finite collection of nonsingular Zariski locally closed subsets of $X$, then there exists an algebraic section of $E$ which is transverse to $X$ and to each $A \in \mathcal{A}$. Indeed, $E$ is generated by some global algebraic sections $s_{1}, \ldots, s_{e}$ (cf. [2, Theorem 12.1.7]), and hence for almost all $\left(t_{1}, \ldots, t_{e}\right) \in \mathbb{R}^{e}$, the algebraic section $t_{1} s_{1}+\cdots+t_{e} s_{e}$ satisfies the required transversality conditions (cf. [7, p. 79, Theorem 2.7]).

Moreover, if $E^{\prime}$ is an algebraic subbundle of $E$, then there exists an algebraic subbundle $E^{\prime \prime}$ of $E$ such that $E=E^{\prime} \oplus E^{\prime \prime}$. To construct $E^{\prime \prime}$, we regard $E$ as an algebraic subbundle of $X \times \mathbb{R}^{m}$ for some $m$ (cf. [2, Definition 12.1.6]). As $E^{\prime \prime}$ we can take the orthogonal complement of $E^{\prime}$ in $E$ with respect to the standard scalar product on $\mathbb{R}^{m}$. 
COROLlary 2.2. Let $X$ be a nonsingular real algebraic variety and let $E$ be an algebraic vector bundle on $X$ of rank $r+1, r \geq 1$. Then there exists a sequence of regular maps

$$
\tilde{X}=X_{r} \stackrel{\pi_{r}}{\longrightarrow} X_{r-1} \stackrel{\pi_{r-1}}{\longrightarrow} \cdots \stackrel{\pi_{2}}{\longrightarrow} X_{1} \stackrel{\pi_{1}}{\longrightarrow} X_{0}=X
$$

such that each $\pi_{i}$ is the blowup of $X_{i-1}$ at a nonsingular subvariety which is either empty or of codimension $r+2-i, 1 \leq i \leq r$, and the pullback vector bundle $\pi^{*} E$ by the composite map $\pi=\pi_{1} \circ \cdots \circ \pi_{r}$ is a direct sum of algebraic line bundles on $X$.

Proof. In view of the remarks preceding Corollary 2.2, the argument used in the proof of Corollary 1.2 is applicable here.

Let $X$ be a compact real algebraic variety. We denote by $H_{d}^{A}(X ; \mathbb{Z} / 2)$ the subgroup of $H_{d}(X ; \mathbb{Z} / 2)$ generated by all homology classes of the form $\varphi_{*}([V])$, where $V$ is a compact $d$-dimensional irreducible nonsingular real algebraic variety, $\varphi: V \rightarrow X$ is a regular map, and $\varphi$ is a birational map between $X$ and the Zariski closure of $\varphi(V)$ in $X$.

REMARK. Although we do not need this here, it is worth while to make some comments. The Zariski closure $W$ of $\varphi(V)$ in $X$ is an irreducible subvariety of $X$ of dimension $d$. The set $W^{0}$ of nonsingular points of $W$ is Zariski open in $W$. Furthermore, the excision property implies

$$
H_{d}(W, W \backslash\{x\} ; \mathbb{Z} / 2) \cong \mathbb{Z} / 2 \quad \text { for every } x \in W^{0} .
$$

Since the regular map $\bar{\varphi}: V \rightarrow W$ defined by $\varphi$ is birational, the image of the homology class $\bar{\varphi}_{*}([V]) \in H_{d}(W ; \mathbb{Z} / 2)$ under the canonical homomorphism

$$
H_{d}(W ; \mathbb{Z} / 2) \rightarrow H_{d}(W, W \backslash\{x\} ; \mathbb{Z} / 2)
$$

generates $H_{d}(W, W \backslash\{x\} ; \mathbb{Z} / 2)$ for all $x$ in a nonempty Zariski open subset of $W^{0}$, and hence by continuity, for all $x \in W^{0}$. There is only one homology class in $H_{d}(W ; \mathbb{Z} / 2)$ with this property (note $\operatorname{dim}\left(W \backslash W^{0}\right)<d$ ); it is called the fundamental class of $W$ and denoted by $[W]$. Thus $\bar{\varphi}_{*}([V])=[W]$, whereas $\varphi_{*}([V]) \in H_{d}^{A}(X ; \mathbb{Z} / 2)$ is the homology class represented by $W$, that is, $\varphi_{*}([V])=i_{*}([W])$, where $i: W \hookrightarrow X$ is the inclusion map.

In fact, every $d$-dimensional subvariety of $X$ carries a unique fundamental class (cf. [3] or [2, Proposition 11.3.1]). The argument above shows

$$
H_{d}^{A}(X ; \mathbb{Z} / 2) \subseteq H_{d}^{\text {alg }}(X ; \mathbb{Z} / 2),
$$

where $H_{d}^{\mathrm{alg}}(X ; \mathbb{Z} / 2)$ is the subgroup of $H_{d}(X ; \mathbb{Z} / 2)$ generated by the homology classes represented by $d$-dimensional subvarieties of $X$. By Hironaka's resolution of singularities theorem [6], this inclusion is an equality.

As an application of Corollaries 1.3 and 2.2, we obtain a very elementary proof (compare with $[3,(5.18)]$ and $[1$, Theorem 2.4]) of the following: 
COROLlary 2.3. Let $X$ be a compact nonsingular real algebraic variety of dimension $n$. If $E$ is an algebraic vector bundle on $X$, then the homology class $D_{X}\left(w_{k}(E)\right)$ is in $H_{n-k}^{A}(X ; \mathbb{Z} / 2)$ for all $k \geq 0$.

Proof. We may assume that $E$ is of constant rank $r+1, r \geq 0$, and $1 \leq k \leq \min \{r+1, n\}$.

First suppose $r \geq 1$, and let $\pi: \widetilde{X} \rightarrow X$ be as in Corollary 2.2. In particular, $\pi^{*} E$ is a direct sum of algebraic line bundles, $\pi^{*} E=L_{1} \oplus \cdots \oplus$ $L_{r+1}$. Hence $w_{k}\left(\pi^{*} E\right)$ is the sum of the cohomology classes of the form $w_{k}(F)$, where $F=L_{i_{1}} \oplus \cdots \oplus L_{i_{k}}, 1 \leq i_{1}<\cdots<i_{k} \leq r+1$. By Corollary 1.3,

$$
D_{X}\left(w_{k}(E)\right)=\pi_{*}\left(D_{\widetilde{X}}\left(w_{k}\left(\pi^{*} E\right)\right)\right)
$$

which implies that $D_{X}\left(w_{k}(E)\right)$ is the sum of the $\pi_{*}\left(D_{\tilde{X}}\left(w_{k}(F)\right)\right)$. It remains to prove that each $\pi_{*}\left(D_{\widetilde{X}}\left(w_{k}(F)\right)\right)$ is in $H_{n-k}^{A}(X ; \mathbb{Z} / 2)$. This can be done as follows. For any $d$-dimensional smooth submanifold $D$ of $\widetilde{X}$, denote by $[D]_{\widetilde{X}}$ its homology class in $H_{d}(\widetilde{X} ; \mathbb{Z} / 2)$. Consider $\widetilde{X}$ as a subvariety of $F$, identifying it with its image by the zero section. Since $F$ is of rank $k$, it is a purely topological fact that

$$
D_{\widetilde{X}}\left(w_{k}(F)\right)=[Z(u)]_{\widetilde{X}}
$$

for any smooth section $u: \widetilde{X} \rightarrow F$ transverse to $\widetilde{X}$. There is a subvariety $Y$ of $X$ such that $\operatorname{dim} Y<\operatorname{dim} X$ and the restriction

$$
\pi \mid \tilde{X} \backslash \pi^{-1}(Y): \tilde{X} \backslash \pi^{-1}(Y) \rightarrow X \backslash Y
$$

is a biregular isomorphism. The subvariety $\pi^{-1}(Y)$ of $\widetilde{X}$ can be expressed as the union of a finite collection $\mathcal{A}$ of nonsingular Zariski locally closed subsets of $\widetilde{X}$. Let $u: \widetilde{X} \rightarrow F$ be an algebraic section of $F$ which is transverse to $\widetilde{X}$ and to each $A \in \mathcal{A}$. Let $V_{1}, \ldots, V_{l}$ be the irreducible components of the nonsingular subvariety $Z(u)$ of $\tilde{X}$. Then

$$
\begin{aligned}
\pi_{*}\left(D_{\widetilde{X}}\left(w_{k}(F)\right)\right) & =\pi_{*}\left([Z(u)]_{\tilde{X}}\right)=\pi_{*}\left(\left[V_{1}\right]_{\widetilde{X}}\right)+\cdots+\pi_{*}\left(\left[V_{l}\right]_{\widetilde{X}}\right) \\
& =\varphi_{1 *}\left(\left[V_{1}\right]\right)+\cdots+\varphi_{k *}\left(\left[V_{l}\right]\right),
\end{aligned}
$$

where $\varphi_{j}=\pi \mid V_{j}: V_{j} \rightarrow X$ for $1 \leq j \leq l$. Note that $V_{j}$ is not contained in $\pi^{-1}(Y)$, and hence $\varphi_{j}$ is a birational map between $V_{j}$ and the Zariski closure of $\varphi_{j}\left(V_{j}\right)$ in $X$. It follows that $\pi_{*}\left(D_{\widetilde{X}}\left(w_{k}(F)\right)\right)$ is in $H_{n-k}^{A}(X ; \mathbb{Z} / 2)$, as required. The proof for $r \geq 1$ is complete.

Now suppose $r=0$, that is, $E$ is a line bundle. Let $s: X \rightarrow E$ be an algebraic section transverse to the zero section. The homology class $D_{X}\left(w_{1}(E)\right)$ is in $H_{n-1}^{A}(X ; \mathbb{Z} / 2)$ since it is represented by the nonsingular subvariety $Z(s)$ of $X$.

There is a version of Corollary 2.3 for $X$ not necessarily compact, with $H_{d}^{A}(X ; \mathbb{Z} / 2)$ defined to be a suitable subgroup of the Borel-Moore homology 
group $H_{d}^{B M}(X ; \mathbb{Z} / 2)$. Such a generalization is an easy exercise for the reader familiar with the Borel-Moore homology.

\section{References}

[1] R. Benedetti and A. Tognoli, Remarks and counterexamples in the theory of real algebraic vector bundles and cycles, in: Géométrie algébrique réelle et formes quadratiques, Lecture Notes in Math. 959, Springer, Berlin, 1982, 198-211.

[2] J. Bochnak, M. Coste and M.-F. Roy, Real Algebraic Geometry, Ergeb. Math. Grenzgeb. 36, Springer, Berlin, 1998.

[3] A. Borel et A. Haefliger, La classe d'homologie fondamentale d'un espace analytique, Bull. Soc. Math. France 89 (1961), 461-513.

[4] W. Fulton, Young Tableaux. With Applications to Representation Theory and Geometry, London Math. Soc. Student Texts 35, Cambridge Univ. Press, Cambridge, 1997.

[5] A. Grothendieck, La théorie des classes de Chern, Bull. Soc. Math. France 86 (1958), $137-154$.

[6] H. Hironaka, Resolution of singularities of an algebraic variety over a field of characteristic zero, I, II, Ann. of Math. 79 (1964), 109-203, 205-326.

[7] M. W. Hirsch, Differential Topology, Grad. Texts in Math. 33, Springer, New York, 1976.

[8] D. Husemoller, Fibre Bundles, McGraw-Hill, New York, 1966.

[9] S. L. Kleiman, Geometry on Grassmannians and applications to splitting bundles and smoothing cycles, Inst. Hautes Études Sci. Publ. Math. 36 (1969), 281-297.

Department of Mathematics and Statistics

University of New Mexico

Albuquerque, NM 87131-1141, U.S.A.

E-mail: kucharz@math.unm.edu 